Article

\title{
Consensus Analysis of Heterogeneous Multi-Agent Systems with Time-Varying Delay
}

\section{Beibei Wang and Yuangong Sun *}

School of Mathematical Sciences, University of Jinan, Jinan 250022, China;

E-Mail: bei219@163.com

* Author to whom correspondence should be addressed; E-Mail: sunyuangong@163.com.

Academic Editor: J. A. Tenreiro Machado

Received: 12 March 2015 / Accepted: 22 May 2015 / Published: 2 June 2015

\begin{abstract}
This paper studies consensus and $H_{\infty}$ consensus problems for heterogeneous multi-agent systems composed of first-order and second-order integrator agents. We first rewrite the multi-agent systems into the corresponding reduced-order systems based on the graph theory and the reduced-order transformation. Then, the linear matrix inequality approach is used to consider the consensus of heterogeneous multi-agent systems with time-varying delays in directed networks. As a result, sufficient conditions for consensus and $H_{\infty}$ consensus of heterogeneous multi-agent systems in terms of linear matrix inequalities are established in the cases of fixed and switching topologies. Finally, numerical simulations are given to illustrate the effectiveness of the theoretical results.
\end{abstract}

Keywords: consensus; $H_{\infty}$ consensus; heterogeneous multi-agent system; linear matrix inequality; time-varying delay

\section{Introduction}

In recent years, the consensus control of multi-agent systems has attracted much attention, due to its broad applications in communication, system control theory, applied mathematics, design of sensor networks, computer science, and so on. Up to now, many researchers have made great achievements for the consensus problem of multi-agent systems by using the method of graph theory, matrix theory, the frequency-domain analysis method, the Lyapunov direct method, etc. [1-5]. Consensus seeking 
has been analyzed mainly for first-order multi-agent systems modeled by a single integrator [6-11] and second-order multi-agent systems modeled by double integrators [12-14], respectively.

Most multi-agent systems considered in the literature are homogeneous, that is all of the agents have the same dynamics behavior. However, the dynamics of the agents is quite different because of various restrictions or the common goals with mixed agents in practical systems. Therefore, it is natural to consider heterogeneous multi-agent system. Compared with the first-order and the second-order integrator multi-agent systems, the study of heterogeneous multi-agent systems composed of both first-order and second-order integrator agents seems to be more complicated. So far, there are very few results on the consensus of heterogeneous multi-agent systems, except Zheng and Wang [15-17], where consensus problems of a heterogeneous multi-agent system were studied by applying the graph theory and the Lyapunov direct method.

Furthermore, in many practical applications involving multi-agent systems, the communication delay [18-23], leader-following networks [24-26], discrete-time [27-29], as well as $H_{\infty}$ consensus problems [30-32] should be considered in the consensus of multi-agent systems. Therefore, in this work, we investigate the consensus and $H_{\infty}$ consensus problems for heterogeneous multi-agent systems in fixed and switching topologies with a time delay.

Because of the complexity of the heterogeneous multi-agent system with time-delays, many existing methods for the consensus of the first-order and the second-order multi-agent systems become invalid. Therefore, the purpose of this paper is to establish consensus and $H_{\infty}$ consensus criteria for heterogeneous multi-agent systems by applying the linear matrix inequality technique. We first rewrite the heterogeneous multi-agent system into a reduced-order system by using a reduced-order transformation. Then, by choosing an appropriate Lyapunov functional, sufficient conditions for consensus are derived in the cases of fixed and switching topologies by using the linear matrix inequality method.

The paper is organized as follows. In Section 2, some preliminaries on graph theory, model formulation and the reduced-order transformation of the heterogeneous multi-agent system are given. In Section 3, sufficient conditions in terms of linear matrix inequalities are established for consensus and $H_{\infty}$ consensus of the heterogeneous multi-agent system with time-varying delay. In Section 4, some simulation results are presented. The conclusion is given in Section 5.

The following notations are used throughout this paper. $\mathcal{I}_{n}=\{1,2, \cdots, n\}$ is an index set. $A^{T}$ means the transpose of the matrix $A$. $I_{n}$ is an $n \times n$-dimensional identity matrix. $\mathbf{a}_{m}=(a, a, \cdots, a)^{T}$ is an $m$-dimensional column vector with $a \in \mathbb{R}$. We say $X>Y$ if $X-Y$ is positive-definite, where $X$ and $Y$ are symmetric matrices of same dimensions. We use an asterisk $*$ to represent a term that is induced by symmetry, and $\operatorname{diag}\{\cdots\}$ stands for a block-diagonal matrix. $L_{2}[0, \infty)$ denotes the space of square-integrable vector functions over $[0, \infty)$.

\section{Preliminaries}

Throughout this paper, we denote a weighted digraph by $\mathcal{G}=(\mathcal{V}, \mathcal{E}, \mathcal{A})$, where $\mathcal{V}=\{1,2, \cdots, n\}$ is the set of nodes with $n \geq 2$; node $i$ represents the $i$-th agent; $\mathcal{E} \subseteq \mathcal{V} \times \mathcal{V}$ is the set of edges; an edge of $\mathcal{G}$ is denoted by an order pair $(i, j)$, and $(i, j) \in \mathcal{E}$ if and only if $a_{j i}>0 ; \mathcal{A}=\left[a_{i j}\right]$ is an $n \times n$-dimensional 
weighted adjacency matrix with $a_{i i}=0$. If $(i, j)$ is an edge of $\mathcal{G}$, node $i$ is called the parent of node $j$. A directed tree is a directed graph, where every node, except one special node without any parent, which is called the root, has exactly one parent, and the root can be connected to any other nodes through paths. The Laplacian matrix $L=\left[l_{i j}\right]$ of digraph $\mathcal{G}$ is defined by $l_{i i}=\sum_{j=1}^{n} a_{i j}$ and $l_{i j}=-a_{i j}$ for $i \neq j$, $i, j \in \mathcal{I}_{n}$.

Suppose that the heterogeneous multi-agent system consists of both first-order and second-order integrator agents, and the number of first-order integrator agents is $m(m<n)$. Then, the heterogeneous multi-agent system is given as follows:

$$
\left\{\begin{array}{l}
\dot{x}_{i}=u_{i}, \quad i \in \mathcal{I}_{m}, \\
\dot{x}_{i}=v_{i}, \quad \dot{v}_{i}=u_{i}, \quad i \in \mathcal{I}_{n} / \mathcal{I}_{m}=\{m+1, m+2, \cdots, n\},
\end{array}\right.
$$

where $x_{i} \in \mathbb{R}$ for $i \in \mathcal{I}_{n}$ and $v_{i} \in \mathbb{R}$ for $i \in \mathcal{I}_{n} / \mathcal{I}_{m}$ are the state of agent $w_{i}$, and $u_{i} \in \mathbb{R}$ for $i \in \mathcal{I}_{n}$ is the control input of agent $w_{i}$, which is also called a protocol throughout this paper.

Consider the following linear protocol:

$$
u_{i}=\left\{\begin{array}{l}
\sum_{j=1}^{n} a_{i j}\left(x_{j}(t-\tau(t))-x_{i}(t-\tau(t))\right), \quad i \in \mathcal{I}_{m}, \\
\sum_{j=1}^{n} a_{i j}\left(x_{j}(t-\tau(t))-x_{i}(t-\tau(t))\right)-k_{i} v_{i}(t), \quad i \in \mathcal{I}_{n} / \mathcal{I}_{m},
\end{array}\right.
$$

where $k_{i}>0, i \in \mathcal{I}_{n} / \mathcal{I}_{m}$, are the feedback gains. The time-delay $\tau$ is the piecewise-continuous function satisfying:

(H1) $0 \leq \tau(t) \leq h$ and $\dot{\tau}(t) \leq d$ for $t \geq 0$, where $h>0$ and $0 \leq d<1$ are constants.

(H2) $0 \leq \tau(t) \leq h$ for $t \geq 0$, where $h>0$ is a constant.

Denote $x(t)=\left(x_{(1)}^{T}(t) \quad x_{(2)}^{T}(t)\right)^{T}$ with $x_{(1)}(t)=\left(x_{1}(t), \cdots, x_{m}(t)\right)^{T}, x_{(2)}(t)=$ $\left(x_{m+1}(t), \cdots, x_{n}(t)\right)^{T}$ and $v(t)=\left(v_{m+1}(t), \cdots, v_{n}(t)\right)^{T}$. Substituting Protocol (2) into System (1), we get the following matrix form of System (1):

$$
\left\{\begin{array}{l}
\dot{x}_{(1)}(t)=-L_{1} x(t-\tau(t)) \\
\dot{x}_{(2)}(t)=v(t) \\
\dot{v}(t)=-L_{2} x(t-\tau(t))-K v(t)
\end{array}\right.
$$

where $K=\operatorname{diag}\left\{k_{m+1}, k_{m+2}, \cdots, k_{n}\right\}, L=\left(L_{1}^{T}, L_{2}^{T}\right)^{T}$ and matrix $L_{1}$ and $L_{2}$ are $m \times n$-dimensional and $(n-m) \times n$-dimensional, respectively.

We introduce the reduced-order transformation as follows:

$$
y_{j}(t)=x_{j+1}(t)-x_{1}(t), \quad j \in \mathcal{I}_{n-1} .
$$

By the straightforward computation, we get:

$$
y_{(1)}(t)=E x_{(1)}(t), \quad x(t)=x_{1}(t) \mathbf{1}_{n}+F y(t),
$$


where $E=\left(\begin{array}{ll}-\mathbf{1}_{m-1} & I_{m-1}\end{array}\right)$ and $F=\left(\begin{array}{ll}\mathbf{0}_{n-1} & I_{n-1}\end{array}\right)^{T}$ are $(m-1) \times m$-dimensional and $n \times(n-1)$-dimensional matrices, respectively, and $y(t)=\left(y_{(1)}^{T}(t) y_{(2)}^{T}(t)\right)^{T}$ with $y_{(1)}(t)=$ $\left(y_{1}(t), \cdots, y_{m-1}(t)\right)^{T}$ and $y_{(2)}(t)=\left(y_{m}(t), \cdots, y_{n-1}(t)\right)^{T}$.

Note that with the fact $L \mathbf{1}_{n}=0$, we can obtain from (3) that:

$$
\dot{y}_{(1)}(t)=E \dot{x}_{(1)}(t)=-E L_{1} F y(t-\tau(t)) .
$$

If $i \in \mathcal{I}_{n-1} / \mathcal{I}_{m-1}$, we have:

$$
\dot{y}_{i}(t)=v_{i+1}(t)-\sum_{j=2}^{n} a_{1 j} y_{j-1}(t-\tau(t)) .
$$

Therefore,

$$
\dot{y}_{(2)}(t)=v(t)-\sum_{j=2}^{n} a_{1 j} y_{j-1}(t-\tau(t))
$$

and:

$$
\dot{v}(t)=-L_{2} F y(t-\tau(t))-K v(t) .
$$

Let $z(t)=\left(y^{T}(t) v^{T}(t)\right)^{T}$; we have the following reduce-order system in matrix form:

$$
\dot{z}(t)=B z(t)+C z(t-\tau(t)),
$$

where:

$$
B=\left(\begin{array}{cc}
0 & 0 \\
0 & I_{n-m} \\
0 & -K
\end{array}\right), \quad C=\left(\begin{array}{cc}
-E L_{1} F & 0 \\
A & 0 \\
-L_{2} F & 0
\end{array}\right)
$$

$A=(\mathbf{a}, \mathbf{a}, \cdots, \mathbf{a})^{T}$ is a $(n-m) \times(n-1)$-dimensional matrix with $(n-1)$-dimensional column vector $\mathbf{a}=\left(-a_{12},-a_{13}, \cdots,-a_{1 n}\right)^{T}$.

When the heterogeneous multi-agent system involves disturbance input, we consider the following protocol of the form:

$$
u_{i}=\left\{\begin{array}{l}
\sum_{j=1}^{n} a_{i j}\left(x_{j}(t-\tau(t))-x_{i}(t-\tau(t))+w_{i j}(t)\right), \quad i \in \mathcal{I}_{m}, \\
\sum_{j=1}^{n} a_{i j}\left(x_{j}(t-\tau(t))-x_{i}(t-\tau(t))-k_{i} v_{i}(t)+w_{i j}(t)\right), \quad i \in \mathcal{I}_{n} / \mathcal{I}_{m},
\end{array}\right.
$$

where $w_{i j}(t) \in L_{2}[0, \infty)$ are disturbance inputs.

Denote the $i$-th row of the matrix $\mathcal{A}$ by $\alpha_{i}, \Sigma_{1}=\operatorname{diag}\left\{\alpha_{1}, \alpha_{2}, \cdots, \alpha_{m}\right\}, \Sigma_{2}=\operatorname{diag}\left\{\alpha_{m+1}, \cdots, \alpha_{n}\right\}$, $w_{i}(t)=\left(w_{i 1}(t), \cdots, w_{i n}(t)\right)^{T}, w(t)=\left(w_{(1)}^{T}(t), w_{(2)}^{T}(t)\right)^{T}$ with $w_{(1)}(t)=\left(w_{1}^{T}(t), \cdots, w_{m}^{T}(t)\right)^{T}$ and $w_{(2)}(t)=\left(w_{m+1}^{T}(t), \cdots, w_{n}^{T}(t)\right)^{T}$.

The matrix form of the heterogeneous multi-agent System (1) with Protocol (6) takes the form:

$$
\left\{\begin{array}{l}
\dot{x}_{(1)}(t)=-L_{1} x(t-\tau(t))+\Sigma_{1} w_{(1)}(t), \\
\dot{x}_{(2)}(t)=v(t) \\
\dot{v}(t)=-L_{2} x(t-\tau(t))+\Sigma_{2} w_{(2)}(t)-K v(t) .
\end{array}\right.
$$


By using the reduced-order transformation (4), we can get the following reduce-order system:

$$
\dot{z}(t)=B z(t)+C z(t-\tau(t))+\tilde{\Sigma} w(t)
$$

where $z(t)$ is defined above, $\tilde{\Sigma}=\left(\begin{array}{cc}E \Sigma_{1} & 0 \\ \Lambda & 0 \\ 0 & \Sigma_{2}\end{array}\right), \Lambda=\left(-\mathbf{a}_{n-m}, 0\right)$ with $\mathbf{a}_{n-m}=\left(\alpha_{1}, \alpha_{1}, \cdots, \alpha_{1}\right)^{T}$ the $(n-m) \times m$-dimensional matrix.

Definition 1. Say System (3) achieves consensus asymptotically, iffor any $x(0)=x_{0}$ and $v(0)=v_{0}$; we have:

$$
\begin{array}{ll}
\lim _{t \rightarrow \infty}\left|x_{i}(t)-x_{j}(t)\right|=0, & i, j \in \mathcal{I}_{n}, i \neq j, \\
\lim _{t \rightarrow \infty} v_{i}(t)=0, & i \in \mathcal{I}_{n} / \mathcal{I}_{m} .
\end{array}
$$

Definition 2. Say System (7) achieves $H_{\infty}$ consensus asymptotically if System (3) achieves consensus asymptotically, and there exists a constant $\gamma>0$, such that:

$$
\int_{0}^{\infty} z^{T}(t) z(t) d t \leq \gamma \int_{0}^{\infty} w^{T}(t) w(t) d t
$$

holds for all nonzero $w \in L_{2}[0, \infty)$ under zero initial condition, where $z(t)=\left(y^{T}(t), v^{T}(t)\right)^{T}$.

\section{Main Results}

The following two lemmas are required.

Lemma 1. [33] (Schur complement). Let $M, P, Q$ be given matrices, such that $Q>0$. Then:

$$
\left(\begin{array}{cc}
P & M \\
M^{T} & -Q
\end{array}\right)<0 \Longleftrightarrow P+M Q^{-1} M^{T}<0
$$

Lemma 2. [20] For any real differentiable vector function $x(t) \in R^{n}$ and any $n \times n$-dimensional constant matrix $W=W^{T}>0$, the following inequality holds:

$$
[x(t)-x(t-\tau(t))]^{T} W[x(t)-x(t-\tau(t))] \leq h \int_{t-\tau(t)}^{t} \dot{x}^{T}(s) W \dot{x}(s) d s, \quad t \geq 0 .
$$

where $0 \leq \tau(t) \leq h$.

Theorem 1. Assume that (H1) holds for given $h>0$ and $0 \leq d<1$. The heterogeneous multi-agent System (3) achieves consensus asymptotically if there exist positive definite matrices $P, Q$ and $R$ of appropriate dimensions, such that:

$$
\left(\begin{array}{ccc}
B^{T} P+P B-Q+R & P C+Q & h B^{T} Q \\
* & -Q-(1-d) R & h C^{T} Q \\
* & * & -Q
\end{array}\right)<0
$$


Proof. Based on the reduced-order transformation, it is sufficient to show that System (5) is asymptotically stable. By Lemma 1, we see that (10) is equivalent to:

$$
\Psi=\left(\begin{array}{cc}
B^{T} P+P B-Q+R & P C+Q \\
* & -Q-(1-d) R
\end{array}\right)+h^{2}(B, C)^{T} Q(B, C)<0 .
$$

Take a Lyapunov functional as follows:

$$
V(t)=z^{T}(t) P z(t)+h \int_{t-h}^{t}(s-t+h) \dot{z}^{T}(s) Q \dot{z}(s) d s+\int_{t-\tau(t)}^{t} z^{T}(s) R z(s) d s .
$$

Denote $\eta^{T}(t)=\left(z^{T}(t), z^{T}(t-\tau(t))\right)$. By (5) and Lemma 2, we have:

$$
\begin{aligned}
\dot{V}(t) \leq & z^{T}(t)\left(B^{T} P+P B\right) z(t)+2 z^{T}(t) P C z(t-\tau(t)) \\
& +h^{2} \eta^{T}(t)(B, C)^{T} Q(B, C) \eta(t) \\
& -[z(t)-z(t-\tau(t))]^{T} Q[z(t)-z(t-\tau(t))] \\
& +z^{T}(t) R z(t)-(1-d) z^{T}(t-\tau(t)) R z(t-\tau(t)) \\
= & \eta^{T}(t) \Psi \eta(t) .
\end{aligned}
$$

By (11), we have that there exists a constant $\alpha>0$, such that $\dot{V}(t) \leq-\alpha\|z(t)\|$. Therefore, the reduced-order System (5) is asymptotically stable, which implies that the heterogeneous multi-agent System (3) achieves consensus asymptotically.

When nothing is known about the derivative, we use the following Lyapunov functional:

$$
V_{1}(t)=z^{T}(t) P z(t)+h \int_{t-h}^{t}(s-t+h) \dot{z}^{T}(s) Q \dot{z}(s) d s
$$

Similar to the proof of Theorem 1, we can easily obtain the following corollary.

Corollary 1. Assume that (H2) holds for given $h>0$. The heterogeneous multi-agent System (3) achieves consensus asymptotically if there exist positive-definite matrices $P$ and $Q$ of appropriate dimensions, such that:

$$
\left(\begin{array}{ccc}
B^{T} P+P B-Q & P C+Q & h B^{T} Q \\
* & -Q & h C^{T} Q \\
* & * & -Q
\end{array}\right)<0 .
$$

Remark 1. For the particular case when $\tau(t)=0$, we have that System (3) achieves consensus asymptotically, if and only if there exists a positive-definite matrix $P \in \mathbb{R}^{(2 n-m-1) \times(2 n-m-1)}$, such that $(B+C)^{T} P+P(B+C)<0$. That is, the $(2 n-m-1) \times(2 n-m-1)$-dimensional matrix $(B+C)$ is Hurwitz.

Remark 2. Similar to the analysis given in [12], the assumption that $\mathcal{G}$ has a spanning tree is only necessary, but not sufficient, for the consensus of System (3). Generally speaking, the consensus of System (3) not only depends on the Laplacian matrix $L(\mathcal{G})$, but also depends on the gain matrix $K$. 
The method used in Theorem 1 can also be applied to the $H_{\infty}$ consensus problem of the heterogeneous multi-agent System (7).

Theorem 2. Assume that (H1) holds for given $h>0$ and $0 \leq d<1$. If there exist positive definite matrices $P, Q$ and $R$ of appropriate dimensions, constants $\alpha>0$ and $\beta>0$, such that:

$$
\left(\begin{array}{cccc}
\Delta & P C+Q & P \tilde{\Sigma} & h B^{T} Q \\
* & -Q-(1-d) R & 0 & h C^{T} Q \\
* & * & -\beta I_{n} & h \tilde{\Sigma}^{T} Q \\
* & * & * & -Q
\end{array}\right)<0
$$

where $\Delta=B^{T} P+P B-Q+R+\alpha I_{2 n-m-1}$, then the heterogeneous multi-agent System (7) asymptotically achieves $H_{\infty}$ consensus with $\gamma=\beta / \alpha$.

Proof. Choose the Lyapunov functional defined by (12). Firstly, (15) implies that (10) holds. By Theorem 1, System (7) with $w=0$ achieves consensus asymptotically. Next, we show that (9) holds with $\gamma=\beta / \alpha$ for all nonzero $w \in L_{2}[0, \infty)$ under zero initial condition. In fact, similar to the analysis given in Theorem 1, we have:

$$
\begin{aligned}
\dot{V}(t) \leq & z^{T}(t)\left(B^{T} P+P B\right) z(t)+2 z^{T}(t) P C z(t-\tau) \\
& +2 z^{T}(t) P \tilde{\Sigma} w(t)+h^{2} \dot{z}^{T}(t) Q \dot{z}(t) \\
& -[z(t)-z(t-\tau)]^{T} Q[z(t)-z(t-\tau)] \\
& +z^{T}(t) R z(t)-(1-d) z^{T}(t-\tau(t)) R z(t-\tau(t)),
\end{aligned}
$$

which implies that:

$$
\dot{V}(t)+\alpha z^{T}(t) z(t)-\beta w^{T}(t) w(t) \leq \xi^{T}\left(\tilde{\Omega}+h^{2} \tilde{\Phi}^{T} Q \tilde{\Phi}\right) \xi
$$

where $\xi=\left(z^{T}(t), z^{T}(t-\tau(t)), w^{T}(t)\right)^{T}$,

$$
\left\{\begin{array}{l}
\tilde{\Phi}=(B, C, \tilde{\Sigma}), \\
\tilde{\Omega}=\left(\begin{array}{ccc}
\Delta & P C+Q & P \tilde{\Sigma} \\
* & -Q-(1-d) R & 0 \\
* & * & -\beta I_{n}
\end{array}\right) .
\end{array}\right.
$$

By (15), (16) and (17), we have that:

$$
\dot{V}(t)+\alpha z^{T}(t) z(t)-\beta w^{T}(t) w(t)<0, \quad t \geq 0 .
$$

Integrating (18) from zero to $\infty$ under zero initial condition, we get:

$$
\int_{0}^{\infty} z^{T}(t) z(t) d t \leq \frac{\beta}{\alpha} \int_{0}^{\infty} w^{T}(t) w(t) d t .
$$

We obtain the following corollary if nothing is known about the derivative of $\tau(t)$. 
Corollary 2. Assume that (H2) holds for given $h>0$. If there exist positive-definite matrices $P, Q$ of appropriate dimensions, constants $\alpha>0$ and $\beta>0$, such that:

$$
\left(\begin{array}{cccc}
\tilde{\Delta} & P C+Q & P \tilde{\Sigma} & h B^{T} Q \\
* & -Q & 0 & h C^{T} Q \\
* & * & -\beta I_{n} & h \tilde{\Sigma}^{T} Q \\
* & * & * & -Q
\end{array}\right)<0
$$

where $\tilde{\Delta}=B^{T} P+P B-Q+\alpha I_{2 n-m-1}$, then the heterogeneous multi-agent System (7) asymptotically achieves $H_{\infty}$ consensus with $\gamma=\beta / \alpha$.

Remark 3. Unlike most of the consensus analysis for multi-agent systems, it does not require that $a_{i j} \geq$ 0 for all $i \neq j$ in the proofs of Theorems 1, 2 and Corollaries 1, 2. Therefore, even when $a_{i j}<0$ for some $i \neq j$, System (7) can also achieve $H_{\infty}$ consensus asymptotically under appropriate conditions.

We now extend the aforementioned results to the following dynamic protocol with switched topologies:

$$
u_{i}=\left\{\begin{array}{l}
\sum_{j=1}^{n} a_{i j}^{\sigma(t)}\left(x_{j}(t-\tau(t))-x_{i}(t-\tau(t))\right), \quad i \in \mathcal{I}_{m}, \\
\sum_{j=1}^{n} a_{i j}^{\sigma(t)}\left(x_{j}(t-\tau(t))-x_{i}(t-\tau(t))\right)-k_{i} v_{i}(t), \quad i \in \mathcal{I}_{n} / \mathcal{I}_{m},
\end{array}\right.
$$

where $t \geq 0, \sigma(t):[0, \infty) \rightarrow \Gamma=\{1,2, \cdots, p\}$ is a switching signal that determines which subsystem is active at time $t ; a_{i j}^{k} \geq 0, i, j \in \mathcal{I}_{n}, k \in \Gamma$, are entries of the weighted adjacency matrix $\mathcal{A}_{k}$. When $\sigma(t)=k \in \Gamma$, we denote the involved digraph by $\mathcal{G}_{k}=\left(\mathcal{V}, \mathcal{E}_{k}, \mathcal{A}_{k}\right)$.

Under the transformation (4), System (1) with Protocol (20) can be described by the following switched system:

$$
\dot{z}(t)=B_{k} z(t)+C_{k} z(t-\tau(t)),
$$

where $z$ is defined above, $B_{k}=\left(\begin{array}{cc}0 & 0 \\ 0 & I_{n-m} \\ 0 & -K\end{array}\right), C_{k}=\left(\begin{array}{cc}-E L_{1}^{k} F & 0 \\ A^{k} & 0 \\ -L_{2}^{k} F & 0\end{array}\right)$,

$$
A^{k}=\left(\begin{array}{cccc}
-a_{12}^{k} & -a_{13}^{k} & \cdots & -a_{1 n}^{k} \\
-a_{12}^{k} & -a_{13}^{k} & \cdots & -a_{1 n}^{k} \\
\vdots & \vdots & \ddots & \vdots \\
-a_{12}^{k} & -a_{13}^{k} & \cdots & -a_{1 n}^{k}
\end{array}\right)
$$

$L_{1}^{k}, L_{2}^{k}$ and $A^{k}$ are defined as $L_{1}, L_{2}$ and $A$, respectively. Let the Lyapunov functionals defined by (12) and (13) be the common Lyapunov functional for the switched System (21), respectively. Then, similar to the analysis in Theorem 1 and Corollary 1, we get the following consensus results for the case of switching topology. 
Theorem 3. Assume that (H1) holds for given $h>0$ and $0 \leq d<1$. The heterogeneous multi-agent System (1) with Protocol (20) can asymptotically achieve consensus under arbitrary switching if there exist positive-definite matrices $P, Q$ and $R$ of appropriate dimensions, such that:

$$
\left(\begin{array}{ccc}
B_{k}^{T} P+P B_{k}-Q+R & P C_{k}+Q & h B_{k}^{T} Q \\
* & -Q-(1-d) R & h C_{k}^{T} Q \\
* & * & -Q
\end{array}\right)<0, \quad k \in \Gamma .
$$

Corollary 3. Assume that (H2) holds for given $h>0$. The heterogeneous multi-agent System (1) with Protocol (20) asymptotically achieves consensus under arbitrary switching if there exist positive definite matrices $P$ and $Q$ of appropriate dimensions, such that:

$$
\left(\begin{array}{ccc}
B_{k}^{T} P+P B_{k}-Q & P C_{k}+Q & h B_{k}^{T} Q \\
* & -Q & h C_{k}^{T} Q \\
* & * & -Q
\end{array}\right)<0, \quad k \in \Gamma .
$$

Consider the following dynamic protocol with disturbance input:

$$
u_{i}=\left\{\begin{array}{l}
\sum_{j=1}^{n} a_{i j}^{\sigma(t)}\left(x_{j}(t-\tau(t))-x_{i}(t-\tau(t))+w_{i j}(t)\right), i \in \mathcal{I}_{m}, \\
\sum_{j=1}^{n} a_{i j}^{\sigma(t)}\left(x_{j}(t-\tau(t))-x_{i}(t-\tau(t))-k_{i} v_{i}(t)+w_{i j}(t)\right), \quad i \in \mathcal{I}_{n} / \mathcal{I}_{m} .
\end{array}\right.
$$

Similar to the proof of Theorem 2, we have the following $H_{\infty}$ consensus results.

Theorem 4. Assume that (H1) holds for given $h>0$ and $0 \leq d<1$. The heterogeneous multi-agent System (1) with Protocol (24) asymptotically achieves $H_{\infty}$ consensus with $\gamma=\beta / \alpha$ under arbitrary switching if there exist positive-definite matrices $P, Q, R$ of appropriate dimensions and constants $\alpha>0$, $\beta>0$, such that:

$$
\left(\begin{array}{cccc}
\Delta_{k} & P C_{k}+Q & P \tilde{\Sigma}_{k} & h B_{k}^{T} Q \\
* & -Q-(1-d) R & 0 & h C_{k}^{T} Q \\
* & * & -\beta I_{n} & h \tilde{\Sigma}_{k}^{T} Q \\
* & * & * & -Q
\end{array}\right)<0, \quad k \in \Gamma,
$$

where $\Delta_{k}=B_{k}^{T} P+P B_{k}-Q+R+\alpha I_{2 n-m-1}, \tilde{\Sigma}_{k}$ is defined as $\tilde{\Sigma} ; B_{k}$ and $C_{k}$ are defined above.

Corollary 4. Assume that (H2) holds for given $0 \leq d<1$. The heterogeneous multi-agent System (1) with Protocol (24) asymptotically achieves $H_{\infty}$ consensus with $\gamma=\beta / \alpha$ under arbitrary switching, if there exist positive-definite matrices $P$ and $Q$ of appropriate dimensions and constants $\alpha>0, \beta>0$, such that:

$$
\left(\begin{array}{cccc}
\tilde{\Delta}_{k} & P C_{k}+Q & P \tilde{\Sigma}_{k} & h B_{k}^{T} Q \\
* & -Q & 0 & h C_{k}^{T} Q \\
* & * & -\beta I_{n} & h \tilde{\Sigma}_{k}^{T} Q \\
* & * & * & -Q
\end{array}\right)<0, \quad k \in \Gamma
$$

where $\tilde{\Delta}_{k}=B_{k}^{T} P+P B_{k}-Q+\alpha I_{2 n-m-1}, \tilde{\Sigma}_{k}$ is defined above. 
Remark 4. In this paper, by introducing a reduced-order transformation, the consensus of heterogeneous multi-agent systems is transformed into the stability of the corresponding delay systems. Therefore, we can derive some other less conservative LMIcriteria by choosing different Lyapunov functionals and using some improved integral inequalities, such as in [34-36].

\section{Numerical Examples}

Consider the following four digraphs with six vertices shown in Figure 1, where Vertices 1-4 denote first-order integrators, Vertices 5-6 denote second-order integrators and the weights associated with the edges shown by solid lines and dashed lines are one and -0.5 , respectively. For the sake of convenience, we let $K=2 I_{2}$.

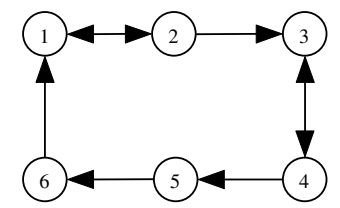

(a)

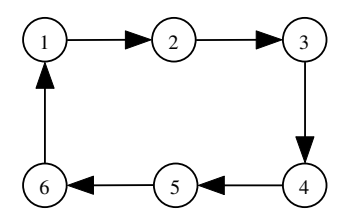

(c)

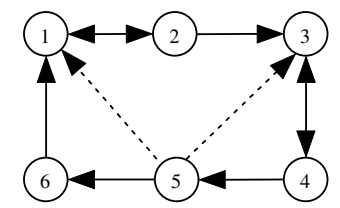

(b)

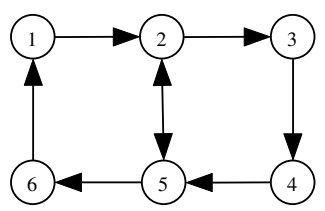

(d)

Figure 1. Four digraphs: (a) $\mathcal{G}_{a} ;(\mathbf{b}) \mathcal{G}_{b} ;(\mathbf{c}) \mathcal{G}_{c} ;(\mathbf{d}) \mathcal{G}_{d}$.

When $\mathcal{G}=\mathcal{G}_{a}$, we have that (14) is feasible for given $0<h \leq 0.3690$. By Corollary 1, System (3) achieves consensus asymptotically. The state trajectory under the stochastic initial condition is shown in Figure 2.

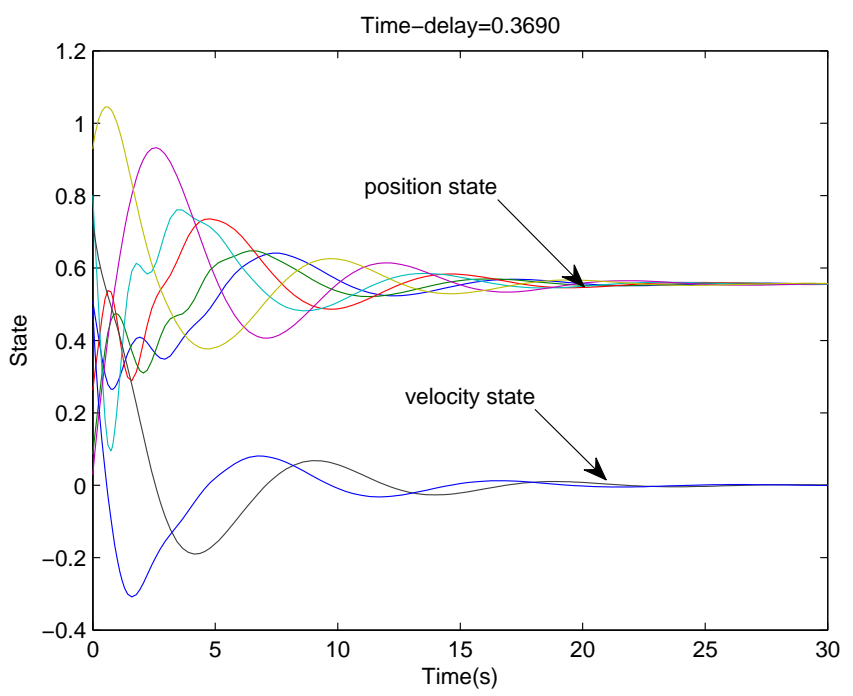

Figure 2. State trajectory of System (3) with $\mathcal{G}=\mathcal{G}_{a}$. 
In Remark 3, we show that Corollary 1 can also be applied to the extreme case when parts of weights $a_{i j}$ are negative. For example, if $\mathcal{G}=\mathcal{G}_{b}$, we have that (14) is still feasible for given $0<h \leq 0.3703$. Therefore, System (3) also achieves consensus asymptotically, even thought there exist negative weights $a_{51}=a_{53}=-0.5$. The corresponding state trajectory under the stochastic initial condition is shown in Figure 3.

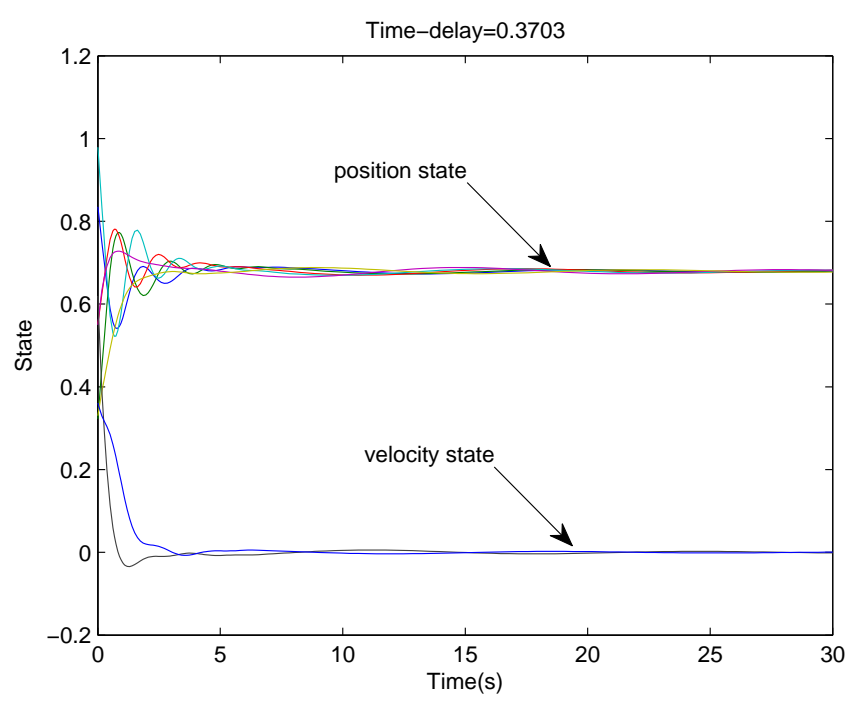

Figure 3. State trajectory of System (3) with $\mathcal{G}=\mathcal{G}_{b}$.

For the case of disturbance input, assume that $\mathcal{G}=\mathcal{G}_{c}$ and $w_{i j}(t)=w_{j}(t)$ for $i, j \in \mathcal{I}_{n}$. Therefore, $\left(\Sigma_{1}^{T} \Sigma_{2}^{T}\right)^{T}=\mathcal{A}$. For given $h=0.3763$, we get from (15) that $\gamma=2.1073$. By Corollary 2, we have that System (7) achieves $H_{\infty}$ consensus with $\gamma=2.1073$. If we let:

$$
w(t)= \begin{cases}(0.1 \sin t) \mathbf{1}, & 0 \leq t \leq 30 \\ 0.0, & \text { otherwise }\end{cases}
$$

the trajectory of System (7) under a stochastic initial condition is shown in Figure 4.

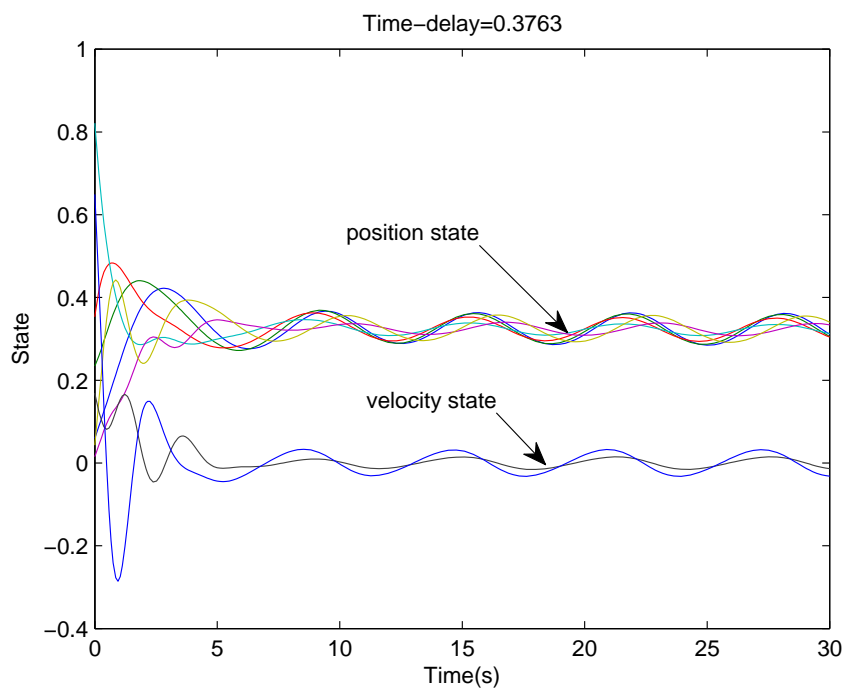

Figure 4. State trajectory of System (7) with $\mathcal{G}=\mathcal{G}_{c}$ and given disturbance. 
For the case of switching topologies $\left\{\mathcal{G}_{c}, \mathcal{G}_{d}\right\}$, we have that (23) is feasible for given $0<h \leq 0.4252$. By Corollary 3, System (1) with Protocol (20) achieves consensus for $0<h \leq 0.4252$ under an arbitrary switching signal. The state trajectories of the system are shown in Figure 5, where the initial condition is stochastic, and the switching signal is periodic, which switches every $T=1$ second according to $\mathcal{G}_{c} \rightarrow \mathcal{G}_{d} \rightarrow \mathcal{G}_{c}$.

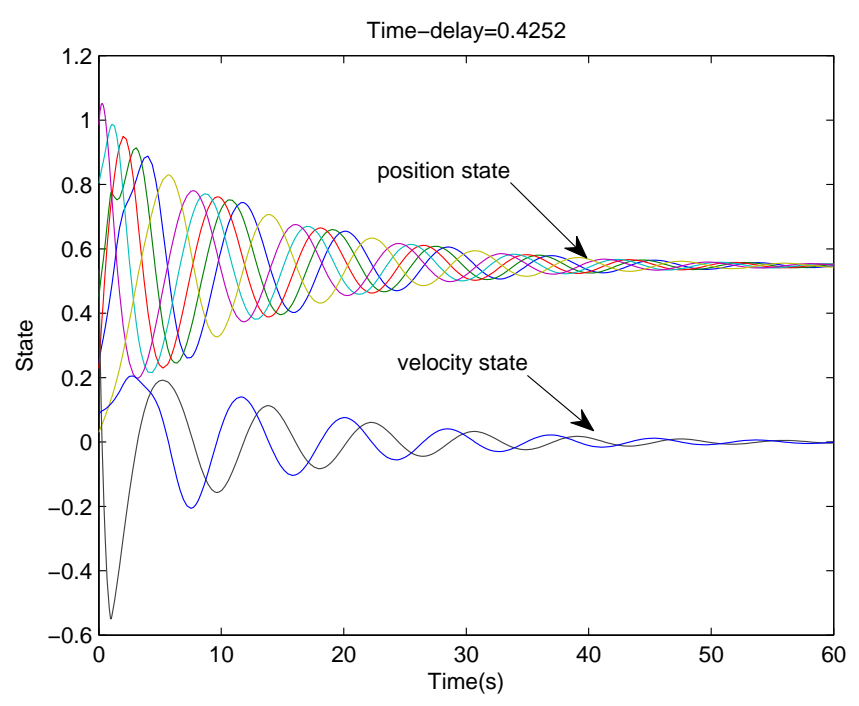

Figure 5. State trajectories of agents under the switching topology.

\section{Conclusions}

In this paper, by using the linear matrix inequality method, we consider the consensus and $H_{\infty}$ consensus problems for heterogeneous multi-agent systems with fixed and switching topologies, as well as time-varying delays. By transforming the heterogeneous multi-agent system into a reduced-order system, sufficient conditions for consensus and $H_{\infty}$ consensus are established in terms of linear matrix inequalities. Finally, simulations are given to illustrate the theoretical results.

\section{Acknowledgments}

This work was supported by the Natural Science Foundation of Shandong Province under Grant No. JQ201119, and the National Natural Science Foundation of China under Grant Nos. 61174217, 61374074 and 61473133.

\section{Author Contributions}

Yuangong Sun designed the research and conceived the idea. Beibei Wang carried out the theoretical analysis and numerical calculations for the results. Both authors have read and approved the final manuscript.

\section{Conflicts of Interest}

The authors declare no conflict of interest. 


\section{References}

1. Moreau, L. Stability of multi-agent systems with time-dependent communication links. IEEE Trans. Autom. Control 2005, 50, 169-182.

2. Olfati-Saber, R.; Fax, J.A.; Murray, R.M. Consensus and cooperation in networked multi-agent systems. Proc. IEEE 2007, 95, 215-233.

3. Ren, W.; Beard, R.W. Distributed Consensus in Multi-Vehicle Cooperative Control; Springer-Verlag: London, UK, 2008.

4. Tan, C.; Liu, G.P. Consensus of networked multi-agent systems via the networked predictive control and relative outputs. J. Frankl. Inst. 2012, 349, 2343-2356.

5. Li, Z.K.; Ren, W.; Liu, X.D.; Xie, L.H. Distributed consensus of linear multi-agent systems with adaptive dynamic protocols. Automatica 2013, 49, 1986-1995.

6. Saber, R.O.; Murray, R.M. Consensus problems in networks of agents with switching topology and time-delays. IEEE Trans. Autom. Control 2004, 49, 1520-1533.

7. Ren, W.; Beard, R.W. Consensus seeking in multiagent systems under dynamically changing interaction topologies. IEEE Trans. Autom. Control 2005, 50, 655-661.

8. Bauso, D.; Giarré, L.; Pesenti, R. Non-linear protocols for optimal distributed consensus in networks of dynamic agents. Syst. Control Lett. 2006, 55, 918-928.

9. Hui, Q.; Haddad, V.M. Distributed nonlinear control algorithms for network consensus. Automatica 2008, 44, 2375-2381.

10. Liu, K.; Xie, G.M.; Ren, W.; Wang, L. Consensus for multi-agent systems with inherent nonlinear dynamics under directed topologies. Syst. Control Lett. 2013, 62, 152-162.

11. Ji, L.H.; Liao, X.F. Consensus problems of first-order dynamic multi-agent systems with multiple time delays. Chin. Phys. B 2013, 22, 50-55.

12. Sun, Y.G.; Wang, L. Consensus problems in networks of agents with double-integrator dynamics and time-varying delays. Int. J. Control 2009, 82, 1937-1945.

13. Li, S.; Du, H.; Lin, X. Finite-time consensus algorithm for multi-agent systems with double-integrator dynamics. Automatica 2011, 47, 1706-1712.

14. Mei, J.; Ren, W.; Ma, G.F. Distributed coordination for second-order multi-agent systems with nonlinear dynamics using only relative position measurements. Automatica 2013, 49, 1419-1427.

15. Zheng, Y.S.; Zhu, Y.; Wang, L. Consensus of heterogeneous multi-agent systems. IET Control Theory Appl. 2011, 5, 1881-1888.

16. Zheng, Y.S.; Wang, L. Consensus of heterogeneous multi-agent systems without velocity measurements. Int. J. Control 2012, 85, 906-984.

17. Zheng, Y.S.; Wang, L. Finite-Time consensus of heterogeneous multi-agent systems with and without velocity measurements. Syst. Control Lett. 2012, 61, 871-878.

18. Xiao, F.; Wang, L. State consensus for multi-agent systems with switching topologies and time-varying delays. Int. J. Control 2006, 79, 1277-1284.

19. Sun, Y.G.; Wang, L. Consensus of multi-agent systems in directed networks with nonuniform time-varying delays. IEEE Trans. Autom. Control 2009, 54, 1607-1613. 
20. Sun, Y.G.; Wang, L.; Xie, G.M. Average consensus in networks of dynamic agents with switching topologies and multiple time-varying delays. Syst. Control Lett. 2008, 57, 175-183.

21. Sun, Y.G. Average consensus in networks of dynamic agents with uncertain topologies and time-varying delays. J. Frankl. Inst. 2012, 349, 1061-1073.

22. Lin, P.; Jia, Y. Multi-Agent consensus with diverse time-delays and jointly-connected topologies. Automatica 2011, 47, 848-856.

23. Lin, P.; Qin, K.; Zhao, H.; Sun, M. A new approach to average consensus problems with multiple time-delays and jointly-connected topologies. J. Frankl. Inst. 2012, 349, 293-304.

24. Hu, J.; Hong, Y. Leader-Following coordination of multi-agent systems with coupling time delays. Physica A 2007, 374, 853-863.

25. Wang, J.; Chen, K.; Ma, Q. Adaptive Leader-Following Consensus of Multi-Agent Systems with Unknown Nonlinear Dynamics. Entropy 2014, 16, 5020-5031.

26. Wang, C.R.; Ji, H.B. Leader-Following consensus of multi-agent systems under directed communication topology via distributed adaptive nonlinear protocol. Syst. Control Lett. 2014, 70, 23-29.

27. Xiao, F.; Wang, L. Consensus protocols for discrete-time multi-agent systems with time-varying delays, Automatica 2008, 44, 2577-2582.

28. Li, Z.K.; Duan, Z.S.; Lewis, F.L. Distributed robust consensus control of multi-agent systems with heterogeneous matching uncertainties. Automatica 2014, 50, 883-889.

29. Yan, Z.P.; Wu, D.; Liu, Y.B. Consensus of Discrete Multiagent System with Various Time Delays and Environmental Disturbances. Entropy 2014, 16, 6524-6538,

30. Ugrinovskii, V. Distributed robust filtering with $H_{\infty}$ consensus of estimates. Automatica 2011, 47, $1-13$.

31. Sun, Y.G.; Wang, L. $H_{\infty}$ consensus of second-order multi-agent systems with asymmetric delays. Syst. Control Lett. 2012, 61, 857-862.

32. Lin, P.; Jia, Y. Robust $H_{\infty}$ consensus analysis of a class of second-order multi-agent systems with uncertainty. IET Control Theory Appl. 2010, 4, 487-498.

33. Boyd, B.; Ghaoui, L.E.; Feron, E.; Balakrishnan, V. Linear Matrix Inequalities in System and Control Theory; SIAM: Philadelphia, PA, USA, 1994.

34. Zhang, X.M.; Han, Q.L. Event-Based $H_{\infty}$ filtering for sampled-data systems. Automatica 2014, $51,55-69$.

35. Zhang, X.M.; Han, Q.L. Robust image filtering for a class of uncertain linear systems with time-varying delay. Automatica 2008, 44, 157-166.

36. Zhang, X.M.; Han, Q.L. New stability criterion using a matrix-based quadratic convex approach and some novel integral inequalities. IET Control Theory Appl. 2014, 8, 1054-1061.

(c) 2015 by the authors; licensee MDPI, Basel, Switzerland. This article is an open access article distributed under the terms and conditions of the Creative Commons Attribution license (http://creativecommons.org/licenses/by/4.0/). 